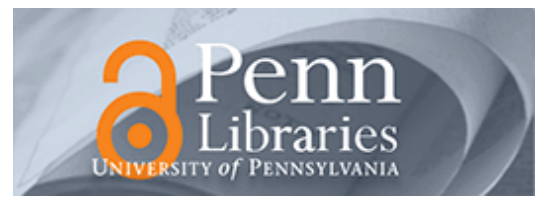

University of Pennsylvania ScholarlyCommons

$11-2018$

\title{
Actuator Transparency and the Energetic Cost of Proprioception
}

\author{
Gavin Kenneally \\ University of Pennsylvania, gake@seas.upenn.edu \\ Wei-Hsi Chen \\ weicc@seas.upenn.edu \\ Daniel Koditschek \\ University of Pennsylvania, kod@seas.upenn.edu
}

Follow this and additional works at: https://repository.upenn.edu/ese_papers

Part of the Electrical and Computer Engineering Commons, and the Systems Engineering Commons

\section{Recommended Citation}

Gavin Kenneally, Wei-Hsi Chen, and Daniel Koditschek, "Actuator Transparency and the Energetic Cost of Proprioception", International Symposium on Experimental Robotics, Buenos Aires . November 2018.

This work was supported by ONR grant \#N00014-16-1-2817, a Vannevar Bush Fellowship held by the last author, sponsored by the Basic Research Office of the Assistant Secretary of Defense for Research and Engineering.

This paper is posted at ScholarlyCommons. https://repository.upenn.edu/ese_papers/862

For more information, please contact repository@pobox.upenn.edu. 


\title{
Actuator Transparency and the Energetic Cost of Proprioception
}

\begin{abstract}
In the field of haptics, conditions for mechanical "transparency"[1] entail such qualities as "solid virtual objects must feel stiff" and "free space must feel free"[2], suggesting that a suitable actuator is able both to do work and readily have work done on it. In this context, seeking actuator transparency has come to mean a preference for minimal dynamics [3] or no impedance [4]. While such general notions seem satisfactory for a haptic interface, actuators with good mechanical transparency are now being used in high-performance robots $[5,6]$ where once again they must be able to do work, but are now also expected to perceive their environment by processing signals related to contact forces in the leg or manipulator when an explicit force sensor is not present. As robotics researchers develop models [7] suitable for programming behaviors that require systematic making and breaking of contact within the environments on which they perform work, actuators must be capable of: (a) generating the high forces at speed needed to accelerate the body during locomotion [5]; (b) robustness to high forces and impacts during locomotion [8]; (c) perceiving high force events quickly, such as touchdown in stance [9]; (d) perceiving contact quickly without exerting significant force on the object, such as in gentle manipulation [10]; and (e) reacting quickly during time-sensitive behaviors [11].
\end{abstract}

This work aims to describe a quantitative assay of transparency that might, for example, predict the advantage in proprioceptive tasks of an electromagnetic directdrive (DD) motor (i.e., one without gearbox), relative to actuation schemes consisting of both a motor and a geared reduction. Specifically, we explore the prospects for characterizing transparency as revealed by comparing the energetic cost of "feeling" the environment. Our sample proprioceptive task is instantiated by a simple torque estimator in Sec. 2. This scheme is then instrumented in simple contact detection experiments paired with a model to empirically explore the relationships between collision energy and detection time delay in Sec. 3 . The actuators are then tested with a feel-cage task to illustrate the advantage of good transparency in Sec. 4.

“For more information: Kod*lab (link to kodlab.seas.upenn.edu)

\section{Keywords}

transparency, contact detection

\section{Disciplines}

Electrical and Computer Engineering | Engineering | Systems Engineering

\section{Comments}

This work was supported by ONR grant \#N00014-16-1-2817, a Vannevar Bush Fellowship held by the last author, sponsored by the Basic Research Office of the Assistant Secretary of Defense for Research and Engineering. 


\title{
Actuator Transparency and the Energetic Cost of Proprioception
}

\author{
Gavin Kenneally ${ }^{\dagger}$, Wei-Hsi Chen ${ }^{\ddagger}$, and Daniel E. Koditschek ${ }^{\ddagger}$
}

\section{Introduction}

In the field of haptics, conditions for mechanical "transparency"[1] entail such qualities as "solid virtual objects must feel stiff" and "free space must feel free"[2], suggesting that a suitable actuator is able both to do work and readily have work done on it. In this context, seeking actuator transparency has come to mean a preference for minimal dynamics [3] or no impedance [4]. While such general notions seem satisfactory for a haptic interface, actuators with good mechanical transparency are now being used in high-performance robots $[5,6]$ where once again they must be able to do work, but are now also expected to perceive their environment by processing signals related to contact forces in the leg or manipulator when an explicit force sensor is not present. As robotics researchers develop models [7] suitable for programming behaviors that require systematic making and breaking of contact within the environments on which they perform work, actuators must be capable of: (a) generating the high forces at speed needed to accelerate the body during locomotion [5]; (b) robustness to high forces and impacts during locomotion [8]; (c) perceiving high force events quickly, such as touchdown in stance [9]; (d) perceiving contact quickly without exerting significant force on the object, such as in gentle manipulation [10]; and (e) reacting quickly during time-sensitive behaviors [11].

This work aims to describe a quantitative assay of transparency that might, for example, predict the advantage in proprioceptive tasks of an electromagnetic directdrive (DD) motor (i.e., one without gearbox), relative to actuation schemes consisting of both a motor and a geared reduction. Specifically, we explore the prospects for characterizing transparency as revealed by comparing the energetic cost of "feeling" the environment. Our sample proprioceptive task is instantiated by a simple torque estimator in Sec. 2. This scheme is then instrumented in simple contact detection experiments paired with a model to empirically explore the relationships between collision energy and detection time delay in Sec. 3. The actuators are then tested with a feel-cage task to illustrate the advantage of good transparency in Sec. 4.

\footnotetext{
This work was supported by ONR grant \#N00014-16-1-2817, a Vannevar Bush Fellowship held by the last author, sponsored by the Basic Research Office of the Assistant Secretary of Defense for Research and Engineering. ${ }^{\dagger}$ Department of Mechanical Engineering and Applied Mechanics, University of Pennsylvania, Philadelphia, PA, USA, gake@seas . upenn. edu. ${ }^{\ddagger}$ Department of Electrical and Systems Engineering, University of Pennsylvania, Philadelphia, PA, USA weicc, kod@seas. upenn.edu
} 


\section{Actuator and Estimator Models}

We quantify four sensorimotor subsystems consisting of an actuator and drive electronics with position sensor [12] built using four actuators denoted U10DD, EC60, T2822, and U10GB, as shown in Table 1. These actuators, offering roughly the same thermally allowable continuous torque, are chosen as representatives of distinctly different robot motor sizing strategies. The T2822 has significant gear reduction $(51.24: 1)$ similar to $[13,14]$ which provides the benefit of increased mass-specific torque and reduced Joule heating at the cost of decreased transparency. The EC60 has a very modest (4.33:1) planetary gearbox, a strategy also employed by the MIT Cheetah [5] and Hermes [15] machines. The U10DD represents the DD strategy employed in manipulators [16] and legged machines, including Minitaur [6] and the Penn Jerboa [12], which benefit from excellent bandwidth and transparency at the cost of increased Joule heating. Our final comparison abandons the uniform continuous torque constraint to explore directly the decreased transparency caused by fitting a small gear reduction (4.33:1) gearbox to a U10.

Table 1 Motor properties

\begin{tabular}{llll|l}
\hline Properties & $\mathrm{U} 10 \mathrm{DD}{ }^{1}$ & $\mathrm{EC} 60^{2}$ & $\mathrm{~T} 2822^{3}$ & $\mathrm{U}^{2} \mathrm{~GB}^{4}$ \\
\hline Physical Properties & & & & \\
Absolute Reduction & $\mathrm{N} / \mathrm{A}$ & $13 / 3$ & $17576 / 343$ & $13 / 3$ \\
Backlash $(\mathrm{deg})$ & 0.0 & 0.6 & 1.0 & 0.6 \\
Mass $m(\mathrm{~kg})$ & 0.405 & 0.730 & 0.251 & 0.865 \\
\hline Performing Work & & & & \\
Max Continuous Torque $\tau_{c}(\mathrm{~N} \cdot \mathrm{m})$ & $\mathbf{2 . 3 6}$ & $\mathbf{1 . 2 1}$ & $\mathbf{2 . 1 2}$ & $\mathbf{1 0 . 2 2}$ \\
Motor Constant $k_{m}(\mathrm{~N} \cdot \mathrm{m} / \sqrt{\mathrm{W}})$ & 0.285 & 0.370 & 0.617 & 1.236 \\
$k_{m} / m(\mathrm{~N} \cdot \mathrm{m} / \sqrt{\mathrm{W}} \cdot \mathrm{kg})$ & 0.705 & 0.506 & 2.56 & 1.430 \\
Nominal Speed $v_{n}(\mathrm{rev} / \mathrm{s})$ & 25.00 & 18.06 & 8.99 & 5.77 \\
Peak Torque $\tau_{p}(\mathrm{~N} \cdot \mathrm{m})$ & 8.05 & $12.35^{*}$ & 11.44 & $35.3^{*}$ \\
Max Continuous Power $P_{c}(W)$ & 92.60 & 34.29 & 29.96 & 92.60 \\
\hline Information Acquisition & & & & \\
Reflected Inertia $J_{r}\left(\mathrm{~kg} \cdot \mathrm{m}^{2}\right)$ & $4.47 \times 10^{-4}$ & $2.27 \times 10^{-3}$ & $3.02 \times 10^{-3}$ & $8.39 \times 10^{-3}$ \\
Static Friction $\tau_{s}(\mathrm{~N} \cdot \mathrm{m})$ & 0.075 & 0.061 & 0.222 & 0.347 \\
Coulomb Friction $\tau_{\text {coul }}(\mathrm{N} \cdot \mathrm{m})$ & 0.032 & 0.026 & 0.114 & 0.253 \\
Viscous Coefficient $b_{v}(\mathrm{~N} \cdot \mathrm{m} /(\mathrm{rad} / \mathrm{s}))$ & 0.099 & 0.153 & 0.835 & 1.890 \\
\hline
\end{tabular}

${ }^{1}$ T-Motor U10 (direct-drive) [6] ${ }^{2}$ Maxon EC60 Flat with Maxon GP42C (1-stage gearbox, 4.33:1) ${ }^{3}$ Turnigy SK3 2822 with Maxon GP32HP (3-stage gearbox, 51.24:1) ${ }^{4}$ T-Motor U10 with GP52C (1-stage gearbox, 4.33:1) ${ }^{*}$ Torque exceeds gearbox rating

\subsection{Properties related to performing work}

Table 1 shows some useful criteria when choosing which motor to perform work. The motor constant, $k_{m}$ [16] is found by the equation $k_{m}=\left(k_{v} \sqrt{R_{m}}\right)^{-1}$, where $k_{v}$ $\left(\frac{\mathrm{rad} / \mathrm{s}}{\mathrm{V}}\right)$ is the motor velocity constant and $R_{m}$ is the motor phase to phase resistance. $k_{m}$ is winding invariant and captures the key trade-off between torque required to work and wasteful Joule heating; for example note the substantial increase in $k_{m}$ relative to U10DD manifesting the greater thermal efficiency resulting from the 
addition of the gearbox in U10GB. $k_{m} / m$ is the mass-normalized motor constant, reflecting the importance of mass budgeting for dynamic machines. Nominal speed $v_{n}$ is calculated at an input voltage of $V_{i n}=15 \mathrm{~V}$ but is not winding invariant, thus this is of limited utility when comparing actuators. The maximum continuous torque is experimentally found by $\tau_{c}=k_{\tau} i_{c}$, where $k_{\tau}\left(\frac{\mathrm{N} \cdot \mathrm{m}}{\mathrm{A}}\right)$ is the motor torque constant and $i_{c}$ is the maximum continuous current, at which the maximum winding temperature reaches $125^{\circ} \mathrm{C}$. As shown in Table 1 , the maximum continuous torque value of the first three actuators are similar enough for our purpose of comparison. Peak torque is also measured experimentally, where stall torque and corresponding motor current $i$ under different PWM duty cycle are measured and fit to the nonlinear torque-current model, $\tau(i)=\tau_{p}-c_{1} e^{-c_{2} i}$, to get the peak torque $\tau_{p}$. It is important to note that the peak torque of EC60 and U10GB exceeds the peak torque rating of their gearboxes, an important consideration for both dynamic maneuvers and longterm wear. Maximum continuous power is calculated at the half point of the motor speed-torque curve, that is $P_{c}=\frac{1}{4} \tau_{c} v_{n}$.

\subsection{Properties related to acquiring information}

While backlash and various frictions all impede backdrivability, reflected inertia is the most critical property to control when designing transparent actuators. With a higher reflected inertia, the actuator is more slowly accelerated by reaction torque from the environment, incurring a longer response time. The inertia of the motors $J_{m}$ and gearbox $J_{g}$ are found in the Maxon catalog[17] or by creating detailed CAD of the rotor. The reflected inertia of a system with a gearbox is calculated as $J_{r}=$ $J_{m} G r^{2}+J_{g}$, where $G r$ is the absolute reduction. Both static friction and backlash act as thresholds for information acquisition since the actuator senses nothing until the torque or deflection (respectively) exceeds a threshold. For purposes of this paper we measure static friction $\tau_{s}$ by hanging incremental weights off a $0.05 \mathrm{~m}$ pulley until the motor starts to turn. By collecting terminal velocities from this mass-hanging task with different hanging masses, the remaining first order (constant and linear) terms characterizing general motor drag $\tau_{d r a g}$ can be fitted as $\tau_{\text {drag }}=\beta_{v} \dot{\theta}+\tau_{\text {coul }}$, as shown in Table 1. For each actuator, there is a very strong linear fit $\left(r^{2} \geq 0.999\right)$. The viscous damping coefficient $b_{v}$ is a collective effect from mechanical Rayleigh damping and the drag-like effect generated from the back emf of the electricalmagnetic motor (the motor controllers are performing sinusoidal voltage control, this term does not exist with ideal current control.) It is clear that $b_{v}$ would contribute more to the energy dissipation as $\tau_{d r a g} \approx b_{v} \dot{\theta}$, and it would require more energy for a system with larger $b_{v}$ to acquire information. Table 1 shows that with the addition of gearboxes, $b_{v}$ increases, thus it would require more energy input to the actuator when acquiring information with a gearbox. 


\subsection{Torque estimation}

We seek to characterize an actuator's ability to estimate torque using a very simple model that depends only on position and ignores all the terms associated with velocity and acceleration, which would require additional sensing or estimation. Assuming a simple DC motor model [17] with negligible inductance, $V=i_{m} R_{m}+G k_{e} \dot{\theta}$, where $V$ is the motor voltage command, $i_{m}$ the current in the motor windings, $k_{e}\left(\frac{\mathrm{v}}{\mathrm{rad} / \mathrm{s}}\right)$ the motor back-emf constant and $G \dot{\theta}$ the motor shaft velocity. Consider a general impedance controller with the form $V=k_{s}^{*}\left(\theta_{d}-\theta_{a}\right)+k_{d}^{*}\left(\dot{\theta}_{d}-\dot{\theta}_{a}\right)$, where $k_{s}^{*}\left(\frac{\mathrm{v}}{\mathrm{rad}}\right)$ and $k_{d}^{*}\left(\frac{\mathrm{v}}{\mathrm{rad} / \mathrm{s}}\right)$ are the proportional and derivative gains respectively. The lowercase " $d$ " and " $a$ " of the motor shaft angle $\theta$ and its velocity $\dot{\theta}$ correspond to the desired and actual readings. The output torque of the actuator is estimated as being proportional to the winding current and scaled by the gear ratio $G, \hat{\tau}=G k_{\tau} i_{m}$, where $k_{\tau}\left(\frac{\mathrm{N} \cdot \mathrm{m}}{\mathrm{A}}\right)$ is the motor torque constant. The estimated torque is

$$
\begin{aligned}
\hat{\tau} & =G k_{\tau} i_{m}=G k_{\tau} \frac{V-G k_{e} \dot{\theta}_{a}}{R_{m}}=\frac{G k_{\tau}}{R_{m}} V-\frac{G^{2} k_{\tau} k_{e}}{R_{m}} \dot{\theta}_{a} \\
& =k_{s}\left(\theta_{d}-\theta_{a}\right)+k_{d}\left(\dot{\theta}_{d}-\dot{\theta}_{a}\right)-\beta \dot{\theta}_{a},
\end{aligned}
$$

where $k_{s}=\frac{k_{s}^{*} k_{\tau} G}{R_{m}}\left(\frac{\mathrm{N} \cdot \mathrm{m}}{\mathrm{rad}}\right), k_{d}=\frac{k_{d}^{*} k_{\tau} G}{R_{m}}\left(\frac{\mathrm{N} \cdot \mathrm{m}}{\mathrm{rad} / \mathrm{s}}\right)$ can are the virtual spring constant and the virtual generalized damper, and $\beta=\frac{G^{2} k_{\tau} k_{e}}{R_{m}}\left(\frac{\mathrm{N} \cdot \mathrm{m}}{\mathrm{rad} / \mathrm{s}}\right)$ can be viewed as the generalized electromechanical drag. Since deriving velocity from position measurements requires some filtering, torque estimation schemes using velocity will inevitably introduce signal delay, further slowing response time. By setting $k_{d}=0$ and assuming $\beta \dot{\theta}$ to be small, the estimate is now much simpler,

$$
\hat{\tau}=k_{s}\left(\theta_{d}-\theta_{a}\right),
$$

yet still accurate enough to be useful in demanding applications of the sort we target such as [9]. Indeed, this is the same approach as used with series-elastic actuators, except the spring is virtual instead of physical.

\section{Contact Detection Experiment, Model, and Data}

To quantify the ability of the motor to acquire information, a contact detection experiment is introduced, where the true contact force measured by a load cell and the estimated contact force acquired from the motor's reading are recorded as well as the detection delay $t_{r}$ and the energy loss $\Delta E$.

\subsection{Contact detection experiment}

We now introduce our proposed transparency measure by relating detection delay, $t_{r}$, to the collision energy required for contact detection. A contact detection ex- 
(a)

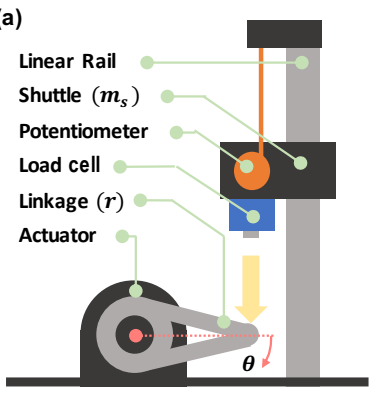

(b)

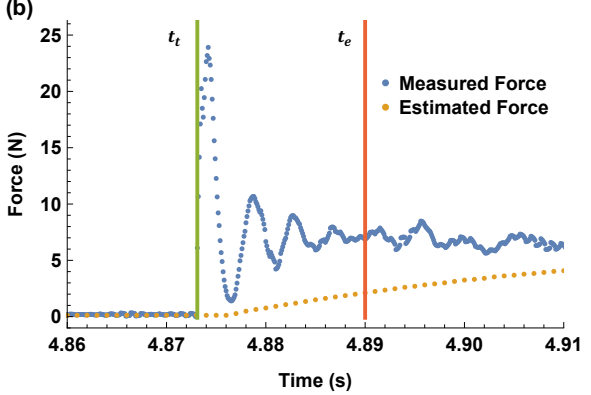

Fig. 1 (a) Setup for the contact detection experiment as described in section 3.1. (b) Single trial of the contact detection experiments in Sec. 3. The green and red vertical lines mark, respectively $t_{t}$, and $t_{e}$, while the blue and yellow dots plot, respectively, $F_{\mu}$ and $F_{\text {est }}$. The linkage length, r, is $0.1 \mathrm{~m}$.

periment was performed for all the actuators, wherein a shuttle of known mass $m_{s}$ installed on an instrumented linear rail is dropped from various heights onto the end of an aluminum link fixed to the actuator output shaft (with overall inertia $J_{m}$, arm length $r$, and actuator angle $\theta$ ), as shown in Fig.1a. The actuator was commanded with impedance $\left(k_{s}=2.0 \frac{\mathrm{N} \cdot \mathrm{m}}{\mathrm{rad}}, k_{d}=0 \frac{\mathrm{N} \cdot \mathrm{m}}{\mathrm{rad} / \mathrm{s}}\right)$. A load cell, sampled at $10 \mathrm{kHz}$ with a DAQ (NI USB-6210), was mounted on a shuttle of known mass $m_{s}(0.54 \mathrm{~kg}$ for U10DD and EC60, $0.71 \mathrm{~kg}$ for T2822 and U10GB). The shuttle was dropped from various heights within the range of $0.03 \mathrm{~m}$ to $1.1 \mathrm{~m}$ onto a $0.1 \mathrm{~m}$ actuator arm. The true contact time $t_{t}$ was recorded when the shuttle hit the actuator arm and the measured force $F_{\mu}$ from the load cell exceeded a threshold of $1 \mathrm{~N} \cdot \mathrm{m}$. A linear potentiometer, sampled at $10 \mathrm{kHz}$, was attached to the shuttle to record the drop distance, which was then used to specify its velocity, $v_{s, t}$, and, in turn, its kinetic energy, $T=\frac{1}{2} m_{s} v_{s, t}^{2}$, at the time of collision, $t_{t}$. When the encoder position error reached a threshold of $0.1 \mathrm{rad}$, chosen by slightly exceeding the deflection of the virtual spring necessary to overcome the actuator with the largest static friction, the time was recorded as the estimated contact $t_{e}$, the velocity of the shuttle mass was marked as $v_{s, e}$, and the estimated contact force $F_{\text {est }}$ was found through Eqn.(1) and the linkage length, r. The motor controller outputs the motor's position at $1 \mathrm{kHz}$, which is synchronized with the DAQ using a digital signal. The detection delay time of the actuator $t_{r}$ is computed as $t_{r}=t_{e}-t_{t}$, and is recorded in each trial in addition to $T, F_{\mu}$, the load cell reading, and $F_{\text {est }}$ as shown in Fig. $1 \mathrm{~b}$.

\subsection{Contact detection model}

Assuming an instantaneous event, we model impact as an inelastic collision between the mass and the actuator $\operatorname{arm}^{5}$. The velocity of the shuttle before the contact is $v_{s, t}$ and the angular velocity of the mass and the actuator after the collision is $\omega_{c}=$ $\frac{m_{s} r v_{s, t}}{J_{m}+m_{s} r^{2}}[18]$. The dynamics after contact can be modeled using the estimated torque

\footnotetext{
${ }^{5}$ This was observed literally in experiments with the more backdrivable actuators; for the highly geared cases (where some repeated bouncing was observer), if contact is not detected on the first collision, it will not be detected in subsequent collision as the first collision necessarily dissipates some energy.
} 

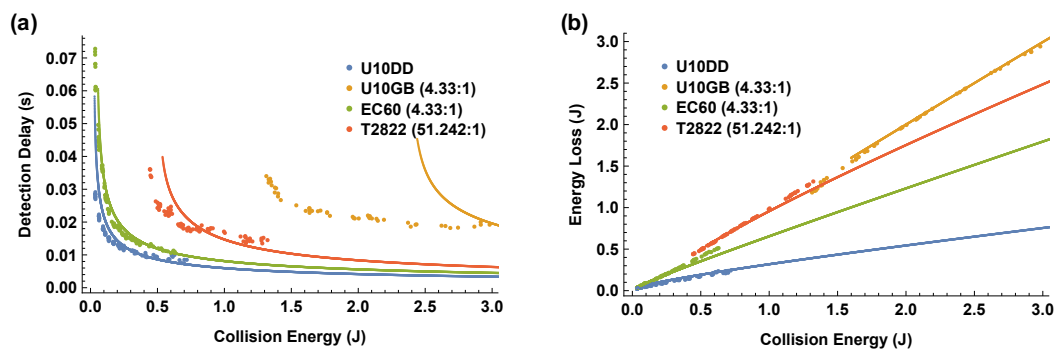

Fig. 2 (a) Detection delay, $t_{r}$, as a function of collision energy, $T$. (b) Energy Loss, $\Delta E$, as a function of collision energy, $T$. The dotted and solid traces plot, respectively, the experimental data and the modeled predictions.

from eqn(1) (with the desired position $\theta_{d}=0$ and the actual position $\theta_{a}=\theta$ ), $\hat{\tau}=$ $-k_{s} \theta$, and the motor general drag $\tau_{d r a g}=\beta_{v} \dot{\theta}+\tau_{\text {coul }}$ as

$$
\left(J_{m}+m_{s} r^{2}\right) \ddot{\theta}=-k_{s} \theta-\beta_{v} \dot{\theta}-\tau_{\text {coul }},
$$

with the initial condition $\theta(0)=0, \dot{\theta}(0)=\omega_{c}$ and has the solution

$$
\theta(t)=e^{\frac{-t\left(\beta_{v}+\Delta\right)}{2 J}}\left(\frac{-\tau\left(\beta_{v}-\Delta\right)-2 J k_{s} \omega_{c}}{2 k_{s} \Delta}\right)+e^{\frac{-t\left(\beta_{v}-\Delta\right)}{2 J}}\left(\frac{-\tau\left(\beta_{v}+\Delta\right)+2 J k_{s} \omega_{c}}{2 k_{s} \Delta}\right)-\frac{\tau_{c o u l}}{k_{s}},
$$

where $\Delta=\sqrt{\beta_{v}^{2}-4 k_{s}\left(J_{m}+m_{s} r^{2}\right)}$. As shown in Fig.1 all these cases represent the condition in which the system is underdamped, so $\Delta$ is a complex number. The trajectories resulting from varied impact velocities, $v_{s, t}$ are solved for until $|\theta(t)| \geq 0.1 \mathrm{rad}$ at time $t:=t_{e}=t_{t}+t_{r}$. With the assumption of inelastic collision, the angular velocity of the shuttle mass and the actuator at the detected time is $\dot{\theta}\left(t_{e}\right)$, and the velocity of the shuttle mass is $v_{s, e}=\dot{\theta}\left(t_{e}\right) r$. Results of the contact detection experiment and the simulation model are shown in Fig. 2.

Fig. 2a shows detection delay $t_{r}$ as a function of collision energy. The experimental results (dots) match well with the numerical simulation from the model (solid curves). From the energy-detection delay curve for each actuator, we can see that it takes more time for the actuator to detect contact when the collision energy is smaller. Because some of the energy transferred to the actuator at the time of collision is converted into motor shaft kinetic energy, it takes more time to rotate the actuator with less energy. However, if the collision results in a force insufficient to overcome the actuator friction then the shaft will not move, precluding any possibility of contact detection, and this accounts for the vertical asymptote of these curves at very low energies. Fig. $2 \mathrm{a}$ also shows that when a motor is equipped with a gearbox, more energy is required to trigger contact detection.

For the actuator to detect contact, there must be enough energy to back-drive the actuator, overcome the frictions, in addition to any Joule heating. This energy can be measured as the energy loss $\Delta E$ in kinetic energy of the shuttle during the detection delay, or 


$$
\Delta E=\frac{1}{2} m_{s}\left(v_{s, t}^{2}-v_{s, e}^{2}\right) .
$$

Fig. $2 \mathrm{~b}$ shows the energy loss as a function of its initial collision energy. $\Delta E$ is the energy transferred from the shuttle into and that back-drives the actuator, and can be thought as the energetic cost of proprioception. The figure shows that as collision energy increases, the energy loss also increases. This is due to the fact that the viscous friction and damping term of the actuator are both proportional to its velocity, thus the actuator takes more energy away when the shuttle has higher relative velocity with the stationary actuator at the beginning of the contact. As predicted by the model, the experiments validate that the larger viscous friction and inertial terms result in a larger slope for the more geared actuators.

\section{Behaviors using Proprioception}

Sensing contact and then re-positioning the actuator is a primitive task embedded in a wide variety of robotics behaviors. We first characterize the performance of these actuators with respect to these tasks, then explore the broader behavioral implications.

\subsection{Feel-cage task}

We illustrate the advantage of good actuator transparency and bandwidth by considering a contact detection and caging task involving balls of varied mass confined to a horizontal rail. Each actuator rotates continuously at $1 \mathrm{rev} / \mathrm{s} \mathrm{CCW}$ and its maximum error accrued during steady operation due to torque ripple [19] and imperfect commutation is recorded as $e_{s}$. The actuator continues to rotate until contact is detected with a position error threshold of $\left(\varepsilon+e_{s}\right)$, where the additional error $\varepsilon$ satisfies the estimated torque error $\tau_{e r r}=k_{s} \varepsilon=0.02 \mathrm{~N} \cdot \mathrm{m}$. If contact is detected, the motor rotates $\mathrm{CW}$ at $10.5 \mathrm{~V}$ open-loop until a position setpoint to kinematically cage the ball. Fig. 3 shows the still image of the feel-cage task performed on the U10DD actuator, and the resulting performance of these actuators with respect to this task with different balls is shown in Table 2. As expected, lighter balls are harder for the actuators to feel then cage since a given collision energy results in faster post-collision ball velocity. The U10DD performs best by successfully detecting contact with and then recirculating in time to feel and cage all of the test balls. As predicted by Fig.2b, the successively more highly geared actuators must impart successively more energy to the balls during collision, propelling them out of the workspace before caging or even detection, in the order predicted by the successively greater slopes of the energy-delay curves. 
Table 2 Feel-cage task results

\begin{tabular}{lllll}
\hline Balls (mass, diameters) $\backslash$ Actuators & U10DD & EC60 & T2822 & U10GB \\
\hline Rubber ball $(0.05 \mathrm{~kg}, 0.063 \mathrm{~m})$ & feel + cage & feel & no detection & no detection \\
Lacrosse ball $(0.145 \mathrm{~kg}, 0.064 \mathrm{~m})$ & feel + cage & feel & no detection & no detection \\
Bocce ball $(0.716 \mathrm{~kg}, 0.073 \mathrm{~m})$ & feel + cage & feel + cage & feel & no detection \\
\hline
\end{tabular}

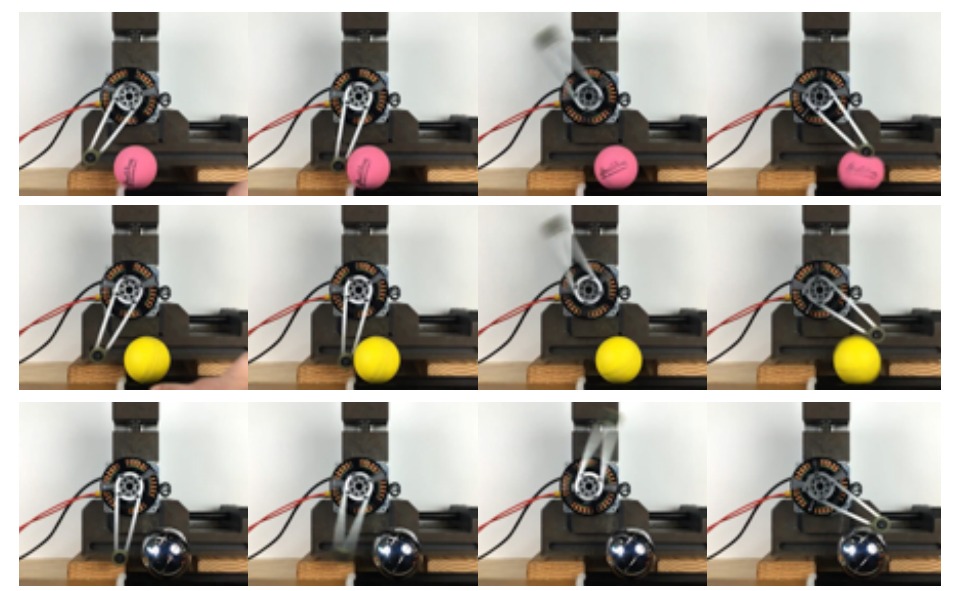

Fig. 3 U10DD performing a feel-cage task for several different balls on a rail. The photo is taken at an interval of $0.05 \mathrm{~s}$

\subsection{Broader behaviors}

The feel-cage task is an example of an emerging class of dynamic primitives that rely on proprioception in which the sensorimotor subsystem must:

1. feel the environment or object of interest using proprioception (causing a delay $t_{\text {feel }}$, or $t_{r}$ in our study, dictated by the transparency)

2. process these signals, and generate a command (causing a delay, $t_{\text {process }}$, dictated by the digital signal processing speed)

3. act as a reaction to the command (causing a delay, $t_{\text {act }}$, dictated by the actuation bandwidth).

The task is successfully accomplished if the total time:

$$
t_{\text {total }}=t_{\text {feel }}+t_{\text {process }}+t_{\text {act }}
$$

is shorter than the time budget, $t_{\text {budget }}$, dictated by the system's dynamics. We now neglect the second step as $t_{\text {process }}$ is typically orders of magnitude faster than the other two (only $1 \mathrm{~ms}$ in our case), and independent of the sensorymotor subsystem.

Dynamic door opening[11] fits this class of behaviors, since the robot jumps up and towards the door with a given horizontal component of velocity (imposing an energy budget). The machine must then catch itself by exerting a normal force on the wall (it is too high off the ground to be supported) and perform the remaining elements of the task before falling back to the ground. The robot must feel the door 
handle with a given limb, then use the same limb to act, first retracting then engaging with the door handle, all before the robot falls back to the ground. If the transparency is too low, the robot will not perceive the door at all with the given energy budget and just fall back to the ground ( $t_{\text {feel }} \geq t_{\text {budget }}$ ). If the transparency is perfect, but the actuation bandwidth is too low, the robot will feel the door, but will not be able to react fast enough to engage the handle before falling to the ground $\left(t_{\text {move }} \geq t_{\text {budget }}\right)$. Finally, any combination of transparency and bandwidth that cause $t_{\text {feel }}+t_{\text {act }}$ to take more time than $t_{\text {budget }}$ (dictated by the dynamics of the robot and the door handle) will also fail.

High speed locomotion[6, 5, 20] also has similar constraints, though the energy budget for the task (in this case related to the vertical component of the velocity) is much higher. The actuator in the leg must feel touchdown without wasting too much energy (plastic collisions and damping in the leg), or consuming too much time. The leg must then act in the remaining stance time to carry out the desired active control (the efficacy of which is dictated by the actuator's bandwidth).

The feel-cage manipulation task can be generalized to consider different kinds of dynamic [21] proprioceptive manipulation in which the manipulator must feel the object before it must then act on the object to do work on in. In this case, the "energy budget," i.e. the threshold of imparted energy beyond which the task will fail, might arise from any combination of physical events, such as:

- pushing the object out of the workspace (or close enough and with the right velocity that the bandwidth is not sufficient to do the move step)

- reaching the manipulator's terminal velocity while contacting the object before it has been sensed, so no further acceleration is possible

- not damaging the object if it is fragile (which can also impose additional constraints on maximum forces).

\section{Conclusion}

We explore transparency in the context of actuation and compare the measure of three different actuators, each broadly representative of a design strategy. Specifically, we explore the use of the energy-delay curves in Fig. $2 b$ as a quantitative assay for the energetic cost of contact detection. Further, we propose a very simple model which broadly captures this trend of transparency by roughly predicting the experimentally revealed energy-delay curves. In turn, this model suggests the possibility of developing a quantitative metric of "transparency" that would predict these models' energy-delay curves from the key parameters presented in Table 1, most notably the reflected inertia, $J_{r}$, and the viscous coefficient $b_{v}$. Such a metric could offer substantial insight when choosing an actuator that will be used to acquire information from the environment. The feel-cage task is an example of an emerging class of dynamic primitives that rely on proprioception in which the actuator must feel the environment, process the information and take action. This simple 1 DoF task inspires various useful higher DoF and highly dynamic tasks such as agile door opening, high speed locomotion, and dexterous manipulation which have 
similar dynamic constraints dictating their success. We are continuing to explore the extent to which similar dynamically derived metrics of transparency may reveal fundamental robotic design limitations.

\section{References}

1. D. Lawrence, L. Y. Pao, M. A. Salada, and A. M. Dougherty, "Quantitative experimental analysis of transparency and stability in haptic interfaces," 1996, pp. 441-449.

2. T. H. Massie and J. K. Salisbury, "The phantom haptic interface: A device for probing virtual objects," in Proceedings of the ASME winter annual meeting, symposium on haptic interfaces for virtual environment and teleoperator systems, vol. 55. Chicago, IL, pp. 295-300.

3. C. R. Carignan and K. R. Cleary, "Closed-loop force control for haptic simulation of virtual environments," 2000.

4. D. A. Lawrence, "Stability and transparency in bilateral teleoperation," IEEE Transactions on Robotics and Automation, vol. 9, no. 5, pp. 624-637, Oct 1993.

5. S. Seok, A. Wang, M. Y. Chuah, D. Otten, J. Lang, and S. Kim, "Design principles for highly efficient quadrupeds and implementation on the mit cheetah robot," in Robotics and Automation (ICRA), 2013 IEEE International Conference on. IEEE, 2013, pp. 3307-3312.

6. G. Kenneally, A. De, and D. Koditschek, "Design principles for a family of direct-drive legged robots," IEEE Robotics and Automation Letters, vol. 1, no. 2, pp. 900-907, 2016.

7. A. M. Johnson, S. A. Burden, and D. E. Koditschek, "A hybrid systems model for simple manipulation and self-manipulation systems," The International Journal of Robotics Research, vol. 35, no. 11, pp. 1354-1392, 2016.

8. S. Kim and P. Wensing, Proprioceptive Actuation Design for Dynamic Legged locomotion, Mar 2016. [Online]. Available: http://meetings.aps.org/Meeting/MAR16/Event/270932

9. A. De and D. E. Koditschek, "Vertical hopper compositions for preflexive and feedbackstabilized quadrupedal bounding, pacing, pronking, and trotting," Int. J. Rob. Res., p. (to appear), 2018.

10. S. Wolf and G. Hirzinger, "A new variable stiffness design: Matching requirements of the next robot generation," in Robotics and Automation (ICRA), 2008 IEEE International Conference on. IEEE, 2008, pp. 1741-1746.

11. T. T. Topping, G. Kenneally, and D. Koditschek, "Quasi-static and dynamic mismatch for door opening and stair climbing with a legged robot," in Robotics and Automation (ICRA), 2017 IEEE International Conference on. IEEE, 2017, pp. 1080-1087.

12. A. De and D. E. Koditschek, "The Penn Jerboa: A platform for exploring parallel composition of templates," arXiv preprint arXiv:1502.05347, 2015.

13. J. A. Grimes and J. W. Hurst, "The design of atrias 1.0 a unique monopod, hopping robot," in Proceedings of the 2012 International Conference on Climbing and Walking Robots and the Support Technologies for Mobile Machines, 2012, pp. 548-554.

14. A. Spröwitz, A. Tuleu, M. Vespignani, M. Ajallooeian, E. Badri, and A. J. Ijspeert, "Towards dynamic trot gait locomotion: Design, control, and experiments with cheetah-cub, a compliant quadruped robot," The International Journal of Robotics Research, vol. 32, no. 8, pp. 932-950, 2013.

15. A. Wang, J. Ramos, J. Mayo, W. Ubellacker, J. Cheung, and S. Kim, "The hermes humanoid system: A platform for full-body teleoperation with balance feedback," in Humanoid Robots (Humanoids), 2015 IEEE-RAS 15th International Conference on. IEEE, 2015, pp. 730-737.

16. H. Asada and K. Youcef-Toumi, Direct-drive robots: theory and practice. MIT press, 1987.

17. Maxon Motors, "Maxon motors program 2017/18," e-catalog, http://maxon.blaetterkatalog. ch/b9990/catalog/index.html?data=b9990/b999045\&lang=e.

18. L. D. Landau and E. M. Lifshitz, Mechanics, 3rd ed. Butterworth-Heinemann, 1982, ch. 4.

19. M. Piccoli and M. Yim, "Anticogging: Torque ripple suppression, modeling, and parameter selection,” The International Journal of Robotics Research, vol. 35, pp. 148-160, 2016. 
20. J. Duperret, B. Kramer, and D. E. Koditschek, "Core actuation promotes self-manipulability on a direct-drive quadrupedal robot," in International Symposium on Experimental Robotics. Springer, 2016, pp. 147-159.

21. N. C. Dafle, A. Rodriguez, R. Paolini, B. Tang, S. S. Srinivasa, M. Erdmann, M. T. Mason, I. Lundberg, H. Staab, and T. Fuhlbrigge, "Extrinsic dexterity: In-hand manipulation with external forces," in 2014 IEEE International Conference on Robotics and Automation (ICRA). IEEE, 2014, pp. 1578-1585. 